\title{
Secretory High-Mobility Group Box 1 Protein Affects Regulatory T Cell Differentiation in Neuroblastoma Microenvironment In Vitro
}

\author{
Thitinee Vanichapol (D), Wararat Chiangjong, ${ }^{2}$ Jirawan Panachan, ${ }^{1}$ \\ Usanarat Anurathapan, ${ }^{1}$ Somchai Chutipongtanate $\mathbb{D}^{2,3}$ and Suradej Hongeng ${ }^{1}$ \\ ${ }^{1}$ Hematology and Oncology Division, Department of Pediatrics, Faculty of Medicine Ramathibodi Hospital, \\ Mahidol University, Bangkok 10400, Thailand \\ ${ }^{2}$ Pediatric Translational Research Unit, Department of Pediatrics, Faculty of Medicine Ramathibodi Hospital, \\ Mahidol University, Bangkok 10400, Thailand \\ ${ }^{3}$ Department of Cancer Biology, University of Cincinnati College of Medicine, Cincinnati, OH 45267, USA \\ Correspondence should be addressed to Somchai Chutipongtanate; schuti.rama@gmail.com
}

Received 19 July 2018; Revised 9 November 2018; Accepted 26 November 2018; Published 16 December 2018

Guest Editor: Jelani Zarif

Copyright (c) 2018 Thitinee Vanichapol et al. This is an open access article distributed under the Creative Commons Attribution License, which permits unrestricted use, distribution, and reproduction in any medium, provided the original work is properly cited.

\begin{abstract}
Neuroblastoma (NB) is the most common extracranial tumor of childhood with poor prognosis in a high-risk group. An obstacle in the development of treatment for solid tumors is the immunosuppressive nature of the tumor microenvironment (TME). Regulatory $\mathrm{T}$ cells (Tregs) represent a $\mathrm{T}$ cell subset with specialized function in immune suppression and maintaining selftolerance. Tregs resident within the tumor milieu is believed to play an important role in immune escape mechanisms. The role of the NB microenvironment in promoting Treg phenotype has never been elucidated. Herein, we demonstrated that the NB microenvironment promoted $\mathrm{T}$ cell activation and one NB cell line, SK-N-SH, manifested an ability to induce Treg differentiation. We identified tumor-derived HMGB1 as a potential protein responsible for Treg phenotype induction. By neutralizing HMGB1, Treg differentiation was abolished. Finally, we adopted a dataset of 498 pediatric NB via the NCBI GEO database, accession GSE49711, to validate clinical relevance of HMGB1 overexpression. Up to $11 \%$ of patients had HMGB1-overexpressed tumors. Moreover, this patient subpopulation showed higher risks of tumor progression, relapse, or death. Our findings emphasize the importance of immunological signature of tumor cells for appropriate therapeutic approach. Upregulation of secretory HMGB1 may contribute to suppression of antitumor immunity through induction of Tregs in the NB microenvironment.
\end{abstract}

\section{Introduction}

Neuroblastoma (NB) is the most common pediatric solid malignancy that has heterogeneity in clinical presentation. Patients with high-risk NB have a dismal prognosis of less than $40 \%$ at five-year survival rate despite intensive therapies [1]. In the past few decades, an approach for adverse prognosis NB patients has shifted toward immunotherapy, i.e., antiGD2 monoclonal antibodies and chimeric antigen receptor (CAR) $\mathrm{T}$ cells. The latter is being tested in a number of clinical trials [2]. However, the efficacy of immunotherapeutic modalities for solid tumors, including NB can be impeded by the immunosuppressive nature of tumor microenvironment (TME) $[3,4]$. In order to improve the potency of immunotherapeutic strategies for NB, a profound understanding of immunosuppressive TME exploited by cancer cells is crucial $[3,4]$.

Tregs represent a small population of $\mathrm{T}$ lymphocytes, normally account for $5-10 \%$ of $\mathrm{CD} 4^{+} \mathrm{T}$ cells [5], and are considered to be a key mediator in maintaining peripheral tolerance. Tregs are comprised of natural Tregs (nTegs), which develop in the thymus, and induced Tregs (iTregs), which are derived from naive $\mathrm{CD} 4^{+} \mathrm{T}$ lymphocytes under the influence of tolerogenic conditions and various factors 
such as IL-10 and TGF- $\beta$ [6]. Both subsets of Tregs are traditionally characterized by expression of the Forkhead Box P3 (Foxp3) transcription factor, which confers suppressive function, and CD25, an activation marker [7]. The difference is that, unlike nTregs, Foxp3 expression of iTregs is relatively unstable [8]. Tregs mediate inhibitory function through multiples mechanisms including secretion of immunosuppressive cytokines (e.g., IL-10 and TGF- $\beta$ ), expression of inhibitory receptors (e.g., CTLA-4, PD-1, and LAG-3), direct cytolysis, metabolic disruption of T effector cells, and induction of tolerogenic dendritic cells (DCs) [7, 9].

The role of Tregs in solid tumors (both intratumoral and peripheral Tregs) and their association with clinical outcomes have remained controversial. On one hand, infiltrating Tregs may play protective roles by controlling inflammation [10]. On the other hand, Tregs may promote tumor progression by suppressing tumor-specific immune responses within the TME [10]. Several studies have evaluated the presence of Tregs in cancer patients [11-17]. The frequency of Tregs is correlated with favorable prognosis in colorectal cancer [11] and head and neck squamous cell carcinoma [12]. In other types of cancer such as breast cancer [13], pancreatic cancer [14], and soft tissue sarcoma [15], high numbers of Tregs have been associated with poor clinical outcome. Although limited data of Tregs in NB patients are available, a few studies found that NB patients had higher Treg frequency $[16,17]$ and therapeutic targeting of Tregs can improve antitumor immunity against NB [1821].

The TME is comprised of cancer cells reside in a specialized niche made up of stromal cells, the vascular system, extracellular matrix, soluble proteins, and infiltrating immune cells including Tregs [3, 4]. A complex array of interactions among cells within the TME is modeled by cancer cells for their own benefits and is facilitated by various growth factors, cytokines, and tumor-derived proteins, i.e., secretomes. The protein composition of cancer secretomes is dynamic as a consequence of genetic mutations and a variety of interactions with external triggers [22]. An analysis of proteins secreted by mouse neuroblastoma showed that these proteins were involved in various aspects of cancer progression including cell proliferation, apoptosis, angiogenesis, and cell adhesion [23]. Although the presence of particular $\mathrm{T}$ cell subsets such as $\mathrm{CD}^{+} \mathrm{T}$ cells or Tregs within tumor lesions has been used as a prognostic factor for decades, the impacts of NB-derived secreted proteins on Treg differentiation within the TME are largely unknown, given that characterization of NB secretomes will give some insights into new therapeutic targeting of Tregs in the NB TME.

In the present study, we demonstrated that a NB cell line SK-N-SH, but not SK-N-AS or SH-SY5Y, produced the permissive TME for Treg differentiation from peripheral blood mononuclear cells (PBMCs). Secretomic profiling, bioinformatics, and functional studies revealed that tumor cell-derived high-mobility group box 1 (HMGB1) protein was a major contributor of Treg differentiation within the NB TME.

\section{Materials and Methods}

2.1. Cell Culture. Three neuroblastoma cell lines, SK-NSH (ATCC HTB-1), SK-N-AS (ATCC CRL-213), and SHSY5Y (ATCC CRL-226), were purchased from the American Type Culture Collection (ATCC; Manassas, VA). SK$\mathrm{N}-\mathrm{SH}$ and SK-N-AS represented neuroblastoma cells with different genetic background. SH-SY5Y cells are a subline of the parental line SK-N-SH with some morphologically distinct phenotypes. All three are MYCN-nonamplified cell lines. Cells were grown in RPMI 1640 medium (Caisson Labs; Smithfield, UT) with 10\% fetal bovine serum (FBS) (Gibco; Fisher Scientific, Waltham, MA) and 1\% penicillin/streptomycin (Gibco).

Human peripheral blood mononuclear cells (PBMCs) were obtained from five healthy donors and were isolated by density gradient centrifugation using Ficoll-Paque (GE healthcare; Little Chalfont, UK). PBMCs were then cultured in RPMI 1640 medium with 10\% fetal bovine serum (FBS), $100 \mathrm{U} / \mathrm{mL}$ IL-2 (PeproTech; Rocky Hill, NJ) and 1\% penicillin/streptomycin. All cells were maintained at $37^{\circ} \mathrm{C}$ with $5 \% \mathrm{CO}_{2}$. This study was approved by the Ethical Clearance Committee on Human Rights Related to Research Involving Human Subjects, Faculty of Medicine Ramathibodi Hospital, Mahidol University (protocol ID 10-60-21).

For coculture experiments, a total number of $10^{5}$ cells of PBMCs were cocultured with each NB cell line at a ratio of 1:3 (PBMC: NB) in complete RPMI 1640 medium with IL-2 $10 \mathrm{U} / \mathrm{mL}$ in 24-well culture plate. Cultures were maintained at $37^{\circ} \mathrm{C} 5 \% \mathrm{CO}_{2}$. On day 4 , the suspension cells were collected and analyzed by flow cytometry. NB cells, which were adherent cells, remained attached to the wells and were excluded from the analysis. PBMC culture without NB cells was served as a blank control.

2.2. Flow Cytometric Analysis. The fluorochrome-coupled monoclonal antibodies (mAbs) used in this study were anti-CD4 phycoerythrin (PE), anti-CD25 PhycoerythrinCyanin 7 (PE-Cy7) and anti-FoxP3 Fluorescein Isothiocyanate (FITC) (\#MHCD0404, \#25-0259-41, \#11-4776-42; ThermoFisher; Florence, KY). The analysis of Treg markers was carried out on day 4 after coculture. Briefly, $10^{5}$ cells of PBMCs were first stained with anti-CD4-PE and anti-CD25$\mathrm{PE}-\mathrm{Cy} 7$ for $30 \mathrm{~min}$ at $4^{\circ} \mathrm{C}$ and washed 2 times in Dulbecco's phosphate buffered saline (DPBS) (GE healthcare) followed by intracellular staining using fixation and permeabilization kit (ThermoFisher). After washing in permeabilization buffer, cells were stained with FoxP3-FITC antibody for another $30 \mathrm{~min}$ at $4^{\circ} \mathrm{C}$ and were then analyzed by $\mathrm{BD}$ FACSVerse flow cytometry with BD FACSuite software (BD Bioscience; San Jose, CA). Tregs were identified as cells with $\mathrm{CD} 4^{+} \mathrm{CD} 25^{+} \mathrm{FoxP}^{+}$using sequential gating strategy.

2.3. Secretome Preparation. For collection of the culture media, SK-N-SH and SK-N-AS cells were plated in T-75 and cultured in complete RPMI 1640 medium until at 80\% confluent. Cells were washed with PBS 4 times to remove serum protein contaminants and then cells were cultured in serum-free RPMI 1640 medium for $24 \mathrm{~h}$. Thereafter, the 
culture supernatants were collected and centrifuged at $1000 \mathrm{~g}$ for $5 \mathrm{~min}$ to remove pellets and cell debris. Secretory proteins in the culture supernatants $(20 \mathrm{~mL}$ per sample) were isolated and concentrated by ultrafiltration using Microsep ${ }^{\mathrm{TM}}$ Advance Centrifugal Devices ( $3 \mathrm{kDa}$ cut-off) (PALL, Port Washington, NY, USA). The 2D Clean-Up Kit (GE healthcare) was used according to the manufacturer's instructions to remove waste products and contaminants that might interfere with further proteomic analysis. The protein pellet obtained from the $2 \mathrm{D}$ Clean-Up kit was resuspended in a lysis buffer containing $7 \mathrm{M}$ urea, $2 \mathrm{M}$ thiourea, $4 \%$ CHAPS, $40 \mathrm{mM}$ Tris, $120 \mathrm{mM}$ dithiothreitol (DTT), $2 \%$ ampholyte $\mathrm{pH} 3-10$, and $1 \%$ protease inhibitor mix. Protein estimation was performed by Bradford assay. The secretome protein samples were stored at $-80^{\circ} \mathrm{C}$ until used.

2.4. Two-Dimensional Gel Electrophoresis (2DE). Secretome samples $(80 \mu \mathrm{g}$ proteins) were mixed with a rehydration buffer (7 M urea, 2M thiourea, 2\% CHAPS, $120 \mathrm{mM}$ DTT, 2\% ampholyte $\mathrm{pH} 3-10$, and bromophenol blue) and rehydrated into a $7 \mathrm{~cm}$ nonlinear immobilized $\mathrm{pH}$ gradient (IPG) strips of $\mathrm{pH}$ range 3-10 (GE healthcare) for 16-18 hours at room temperature. The first dimension separation (or isoelectric focusing) was performed by the Ettan IPGphor III IEF System (GE healthcare) at $20^{\circ} \mathrm{C}$ in a stepwise voltage increase to reach $8,333 \mathrm{Vhrs}$ with the limited of $50 \mu \mathrm{A} /$ strip. After isoelectric focusing, the strips were equilibrated in the first equilibration buffer (6 M Urea, $112 \mathrm{mM}$ Tris- $\mathrm{HCl}$ ( $\mathrm{pH}$ 8.8), 30\% glycerol, $4 \%$ SDS, and $130 \mathrm{mM} \mathrm{DTT}$ ) for $15 \mathrm{~min}$, followed by the second equilibration buffer containing the same compositions with $135 \mathrm{mM}$ iodoacetamide (IAA) instead of DTT for $15 \mathrm{~min}$. The second dimensional analysis was performed on $12.5 \%$ SDSPAGE using SE260 mini-Vertical Electrophoresis Unit at 150 $\mathrm{V}$ for approximately $2 \mathrm{~h}$. Staining of $2 \mathrm{DE}$ gels was performed using Coomassie Colloidal Blue G-250 staining.

2.5. Spot Quantification. Gels were scanned by ImageScanner III (GE Healthcare) and the images ( $\mathrm{n}=5$ secretome samples per cell line) were analyzed using Image master 2D platinum software version 7.0 (GE healthcare). Normalization of each protein spot was carried out in relation to the total protein spot intensity in the same gel image. Analysis of the expression level of the protein spots was performed by Student's t-test. The protein spots that passed the threshold of fold-change $>3$ and $p$-value of $<0.05$ were considered significant and were subjected to protein identification by mass spectrometry.

2.6. Mass Spectrometry. Protein spots of interest were excised from the 2DE gels, washed with deionized (DI) water, destained using $50 \mathrm{mM} \mathrm{NH} \mathrm{NHCO}_{3}$ in $50 \%$ acetonitrile $(\mathrm{ACN})$ at $37^{\circ} \mathrm{C}$ for $15 \mathrm{~min}$, and dried in a SpeedVac concentrator. The gel pieces were reduced by incubating in $10 \mathrm{mM}$ DTT/100 $\mathrm{mM} \mathrm{NH}_{4} \mathrm{HCO}_{3}$ at $56^{\circ} \mathrm{C}$ for $30 \mathrm{~min}$. The reduced gel pieces were then washed with $\mathrm{ACN}$ and then dried with SpeedVac concentrator and were alkylated with $55 \mathrm{mM}$ IAA/100 $\mathrm{mM}$ ammonium bicarbonate at room temperature for $30 \mathrm{~min}$ in the dark. The alkylated gel piece was washed with ACN and all liquid was removed using SpeedVac concentrator. The proteins were then digested by incubating $18 \mathrm{~h}$ at $37^{\circ} \mathrm{C}$ with trypsin (Promega Corporation, Madison, WI, USA). The trypsin reaction was stopped by adding $10 \mu \mathrm{l}$ of $5 \%$ trifluoroacetic acid (TFA)/ACN (ratio 1:2). The gel piece in solution was vigorously vortexed for $1 \mathrm{~min}$ before collecting the protein mixtures into a new collection tube and then dried by a SpeedVac.

The dried peptides were resuspended with $2 \mu \mathrm{l}$ of 0.1\%TFA and spotted on MTP 384 target plate ground steel BC (Bruker Daltonik GmbH, Breman, Germany) and mixed with $2 \mu \mathrm{l}$ of $2.5 \mathrm{mg} / \mathrm{ml}$ alpha-cyano-4-hydroxycinnamic acid matrix substance in $80 \%$ ACN/0.1\%TFA. After drying peptide spot solution, the target plate was operated on Ultraflex II MALDI-TOF/TOF (Bruker) mass spectrometer. The parent ions in each sample were detected at positive refractor mode with m/z 700-3500 Da, frequency $2000 \mathrm{~Hz}$, $2.3 \mathrm{mV}$ analog offset. Parent ions with signal to noise ratio more than 3 were further fragmented to transition ions in LIFT mode. Mass of parent ions and transition ions were combined into one.data file and search via Mascot server by using Biotool software (Bruker). Mascot search parameters included carbamidomethylation at cysteine residue for fixed modification, oxidation at methionine for variable modification, monoisotopic ion, $\pm 200 \mathrm{ppm}$ for peptide tolerance, \pm 0.5 $\mathrm{Da}$ for fragment ion tolerance, Swiss-Prot database, Homo sapiens taxonomy, $1+$ charge state, and trypsin digestion with 1 missed cleavage allowed. Identified proteins were provided ions scores $(>28)$ more than identity threshold and contained at least one significant peptide.

2.7. Western Blot. Secretome samples ( $20 \mu \mathrm{g}$ protein) were resolved in $12.5 \%$ SDS-PAGE gel and proteins were electrotransferred onto a nitrocellulose membrane (Merck Millipore, Burlington, MA). After blocking with 5\% skim milk, the membrane was incubated with primary antibody, anti-HMGB1 (1:1000 dilution; Biolegend, San Diego, $\mathrm{CA}$,) overnight at $4^{\circ} \mathrm{C}$. After washing, the membranes were incubated with secondary antibody, goat anti-mouse immunoglobulins/HRP (1:2000 dilution; Dako, Santa Clara, CA) for $1 \mathrm{~h}$. The membrane was then incubated with enhanced chemiluminescence reagent (GE Healthcare) and the immunoreactive bands were visualized by $\mathrm{X}$-ray film exposure. Anti-GAPDH (1:5000 dilution; Abcam, Cambridge, UK) was used as a loading control with the same secondary antibody (1:10000 dilution; Dako).

2.8. Neutralization Assay. To validate a functional role of HMGB1 in Treg differentiation during the coculture of PBMCs and SK-N-SH cells, varied concentrations of antiHMGB1 antibody $(1 \mu \mathrm{g} / \mathrm{mL}, 2 \mu \mathrm{g} / \mathrm{mL}$, or $3 \mu \mathrm{g} / \mathrm{mL})$ and IgG isotype control $(1 \mu \mathrm{g} / \mathrm{mL})$ (Dako) were added to the coculture experiment as aforementioned. The suspended cells were collected and analyzed by flow cytometry on day 4 .

To confirm that secreted HMGB1 in the secretomes, but not factors associated with direct cell-cell contact, play roles in Treg differentiation, the culture media containing SK$\mathrm{N}-\mathrm{SH}$ secretomes with the supplements of $10 \% \mathrm{FBS}$ and 
IL-2 $10 \mathrm{U} / \mathrm{mL}$ was used to treat $10^{5}$ cells of PBMCs in the presence or absence of anti-HMGB1 antibody $(1 \mu \mathrm{g} / \mathrm{mL}, 2$ $\mu \mathrm{g} / \mathrm{mL}$, or $3 \mu \mathrm{g} / \mathrm{mL})$ or IgG isotype control $(1 \mu \mathrm{g} / \mathrm{mL})$. On day 4 , the treated cells were analyzed by flow cytometry. PBMCs cultured in complete RPMI 1640 medium with IL-2 $10 \mathrm{U} / \mathrm{mL}$ was used as a negative control. This experiment was performed in 3 biological replicates.

2.9. Statistical and Data Analysis. Data analysis was performed by Excel and R package MetaboAnalystR (https:// www.metaboanalyst.ca) [24]. The results were expressed as mean \pm SD. Statistical analysis was performed by $\mathrm{t}-$ test or ANOVA with Tukey HSD post hoc as appropriate. All experiments were carried out as 5 biological replicates unless otherwise stated. $P$-value $<0.05$ was considered as statistically significant. A self-organized heatmap was based on Euclidean distance and average linkage. Functional annotation of significant proteins was performed by STRING (https://www.string-db.org) [25], Panther (http://www.pantherdb.org) [26], and David functional annotation (https://david.ncifcrf.gov) [27].

The Gene Expression Omnibus (GEO) (https://www.ncbi .nlm.nih.gov/geo) [28], accession GSE49711, was accessed to adopt HMGB1 mRNA expression in tumors (from RNA-seq) and clinical data of 498 patients previously reported as part of the MicroArray Quality Control-III/Sequencing Quality Control (MAQC-III/SEQC) study [29]. HMGB1 mRNA upregulation and downregulation were defined as the $\mathrm{z}$ score of $>1.25$ and $<-1.25$, respectively. Associations between HMGB1 mRNA alterations and clinical outcomes (i.e., the occurrence of events and death) were determined by logistic regression and presented as the adjusted odds ratio (OR) with 95\% confidence interval (CI) using R package "epiDisplay" (available via http://www.cran.r-project.org). MYCN amplification, a strong prognostic factor of neuroblastoma, was used to adjust the influence of HMGB1 mRNA alterations on clinical outcomes. $\mathrm{P}$-value $<0.05$ was considered as statistical significance.

\section{Results}

3.1. Coculture of PBMCs with NB Cell Lines Showed Phenotypic Differentiation of $\mathrm{CD}^{+}{ }^{+} \mathrm{T}$ Cell Subsets. To investigate whether NB cell lines could generate a permissive microenvironment that mediated $\mathrm{T}$ cell phenotypic changes toward Treg differentiation, three NB cell lines, SK-N-SH, SK-N-AS, and SH-SY5Y were cocultured with PBMCs $(n=5$ healthy individuals) for 4 days and then analyzed for Treg markers by flow cytometry. The sequential gating strategy for Treg enumeration is presented in Figure 1(a). At baseline, PBMCs culture alone showed $40.5 \pm 7.1 \%$ of $\mathrm{CD} 4^{+} \mathrm{T}$ lymphocytes (Figure $1(\mathrm{~b})$ ), $11.4 \pm 1.0 \%$ of activated $\mathrm{CD} 4^{+} \mathrm{CD} 25^{+} \mathrm{T}$ cells (Figure $1(\mathrm{c})$ ), and $8.6 \pm 0.9 \%$ of $\mathrm{CD} 4^{+} \mathrm{CD} 25^{+}$Foxp $3^{+}$Tregs (Figure $1(\mathrm{~d})$ ), respectively. After being cocultured with three NB cell lines, the percentage of $\mathrm{CD} 4{ }^{+} \mathrm{T}$ cells remained unchanged (Figure 1(b)); however, there were significant increases in the percentage of $\mathrm{CD} 4^{+} \mathrm{CD} 25^{+} \mathrm{T}$ lymphocytes (up to $20 \%$ ) in all cocultured conditions (Figure 1(c)). Interestingly, only
PBMCs coculture with SK-N-SH cells showed significantly higher number of $\mathrm{CD}^{+} \mathrm{CD} 25^{+} \mathrm{Foxp}^{+}$Tregs as compared to the PBMCs culture alone $(13.07 \pm 1.22 \%$ versus $8.64 \pm 0.85 \%$, $p<0.001$ ) (Figure $1(d)$ ), suggesting that some particular factors resided in the TME of SK-N-SH cells could augment Treg differentiation.

3.2. Analysis of Protein Differential Expression in SK-N-SH versus SK-N-AS Secretomes. It is hypothesized that Treg differentiation was mediated by unique molecules that only presented in SK-N-SH-derived secretomes. To elucidate this hypothesis, a comparison of secretomic profiles of SK-N-SH versus SK-N-AS was performed by proteomic analysis. We chose SK-N-AS over SH-SY5Y (a subline of SK-N-SH) since this cell line has a different genetic abnormality background to SK-N-SH. Representative gel images of the SK-N-SH and $\mathrm{SK}-\mathrm{N}$-AS secretomic profiles (5-independent samples/group) are shown in Figure 2. A total of 30 significant protein spots were detected at the threshold of fold-change of $>3$ and $p$ value of $<0.05$ between groups (Figure 2) and were analyzed by LC-MS/MS for 29 unique protein identities (Table 1 and Supplementary Table 1).

3.3. Protein Bioinformatics Revealed Secretory HMGB1 May Involve in Treg Differentiation. The significant proteins from proteomic analysis were then annotated by bioinformatic tools to pinpoint the unique proteins that potentially mediated Treg differentiation in the NB TME. Figure 3(a) showed the relative abundance of 29 significantly altered proteins between SK-N-SH and SK-N-AS secretomes in a selforganized heatmap, in which two major clusters of 23 upregulations and 6 downregulations were detected. We considered that Treg-inducible proteins in SK-N-SH-derived secretomes should belong to the upregulation cluster. STRING protein network with GO-term cellular component analysis showed that 24 proteins were enriched in extracellular compartments, representing cell secretory products (Figure 3(b) and Supplementary Table 2). PANTHER protein classification showed that a large proportion of the identified proteins were chaperones, whereas there were 2 proteins that belong to the class of signaling molecule, SPARC and HMGB1 (Figure 3(c) and Supplementary Table 3). David functional annotation showed that, of all 29 significant proteins, only HMGB1 may involve in the regulation of $\mathrm{T}$ cell response to tumor cells (GO:0002840) (Figure 3(d) and Supplementary Table 4). Based on bioinformatic analyses, a literature review focusing on HMGB1 was performed. Since previous studies supporting its potential effects on Treg immunomodulation [30, 31], we chose HMGB1 as a candidate protein for further studies. Before functional validation, Western immunoblot analysis was used to confirm HMGB1 protein expression in secretomic samples. As expected, SK-N-SH-derived secretomes contained a higher level of HMGB1 as compared to those of SK-N-AS (Figure 3(e)).

3.4. Neutralization of Secretory HMGB1 Suppressed Treg Differentiation in the NB TME. To elucidate whether HMGB1 in 

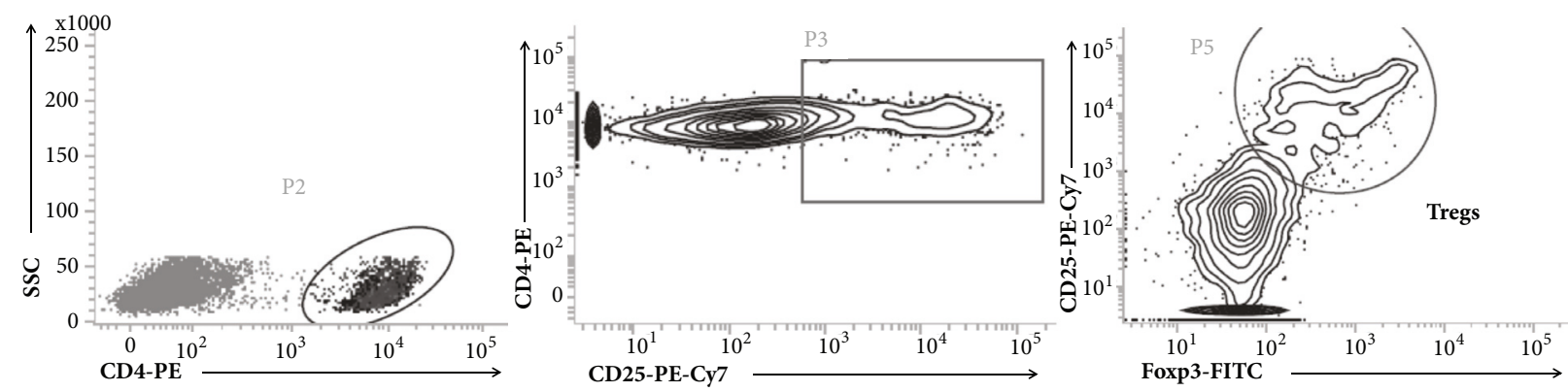

(a)

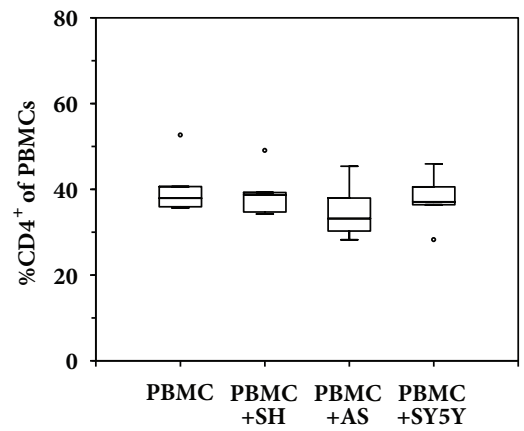

(b)

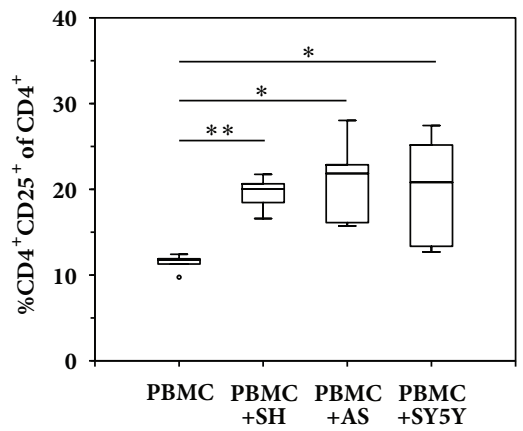

(c)

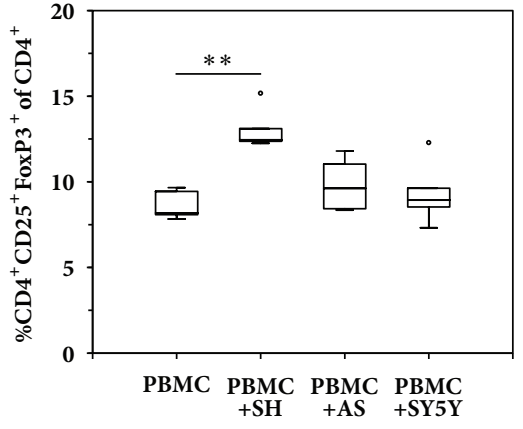

(d)

FIgURE 1: The expression of phenotypic Treg markers in coculture experiments by flow cytometry. PBMCs obtained from 5 healthy donors were cultured in the absence (PBMC) or presence of SK-N-SH (PBMC+SH), SK-N-AS (PBMC+AS), and SH-SY5Y (PBMC+SY5Y) for 4 days prior to flow cytometric analysis. (a) The sequential gating strategy of Treg enumeration. (b) The percentage of CD4 ${ }^{+} \mathrm{T}$ cells of PBMCs. (c) The percentage of $\mathrm{CD} 4^{+} \mathrm{CD} 25^{+}$activated T cells of total $\mathrm{CD} 4^{+} \mathrm{T}$ cells. (d) The percentage of $\mathrm{CD} 4^{+} \mathrm{CD} 25^{+}$Foxp $3^{+}$Treg cell of total CD $4^{+} \mathrm{T}$ cells. The results showed that the PBMC+SH coculture condition, but not those of PBMC+AS or PBMC+SY5Y, had significant increase in the Treg frequency as compared to the control condition (PBMC), suggesting that a particular microenvironment of SK-N-SH cells mediated Treg differentiation. Values represent mean \pm SD of 5 independent experiments $\left({ }^{*} p<0.05 ;{ }^{* *} p<0.001\right)$.
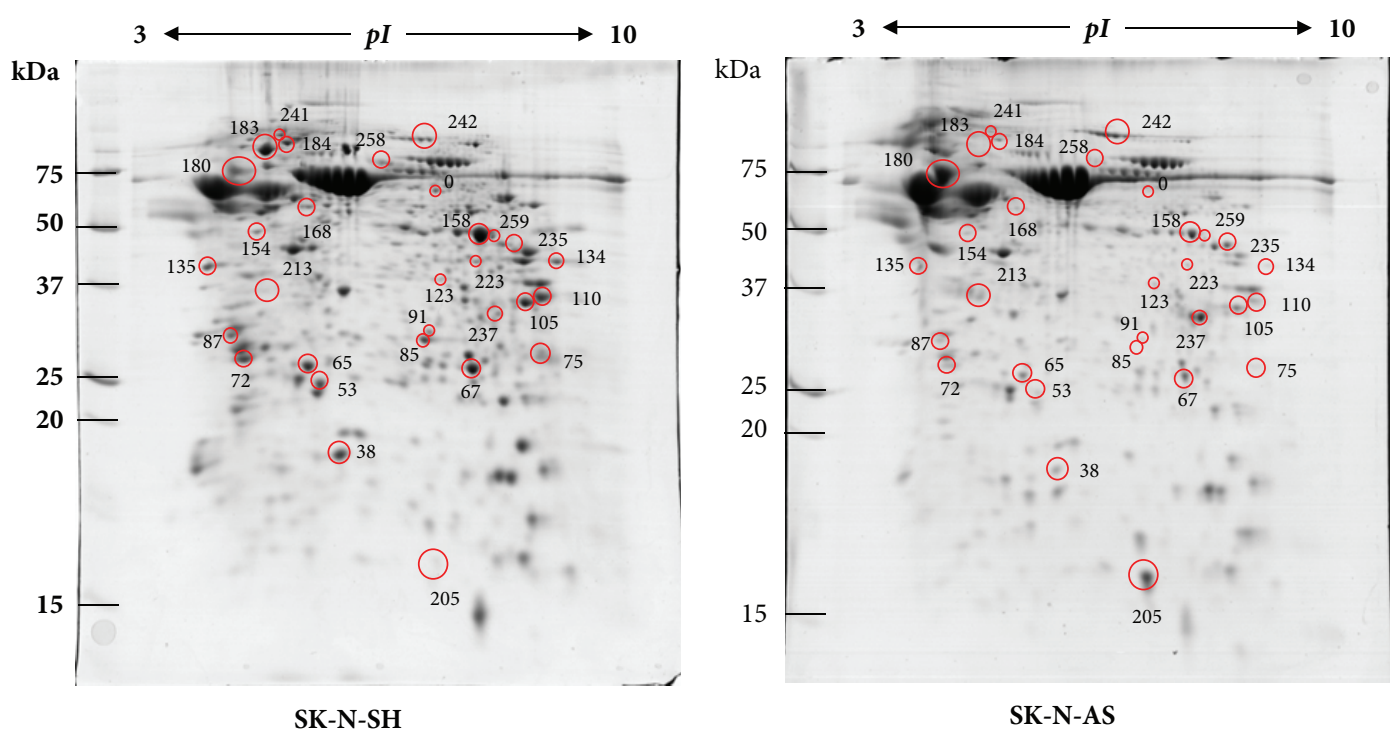

FIGURE 2: Representative 2DE gel images showing differentially expressed proteins in SK-N-SH versus SK-N-AS secretomes ( $\mathrm{n}=5$ per group). Thirty significantly altered protein spots were labeled by red circles with spot ID in which their corresponding data were reported in Table 1 and Supplementary Table 1 . 


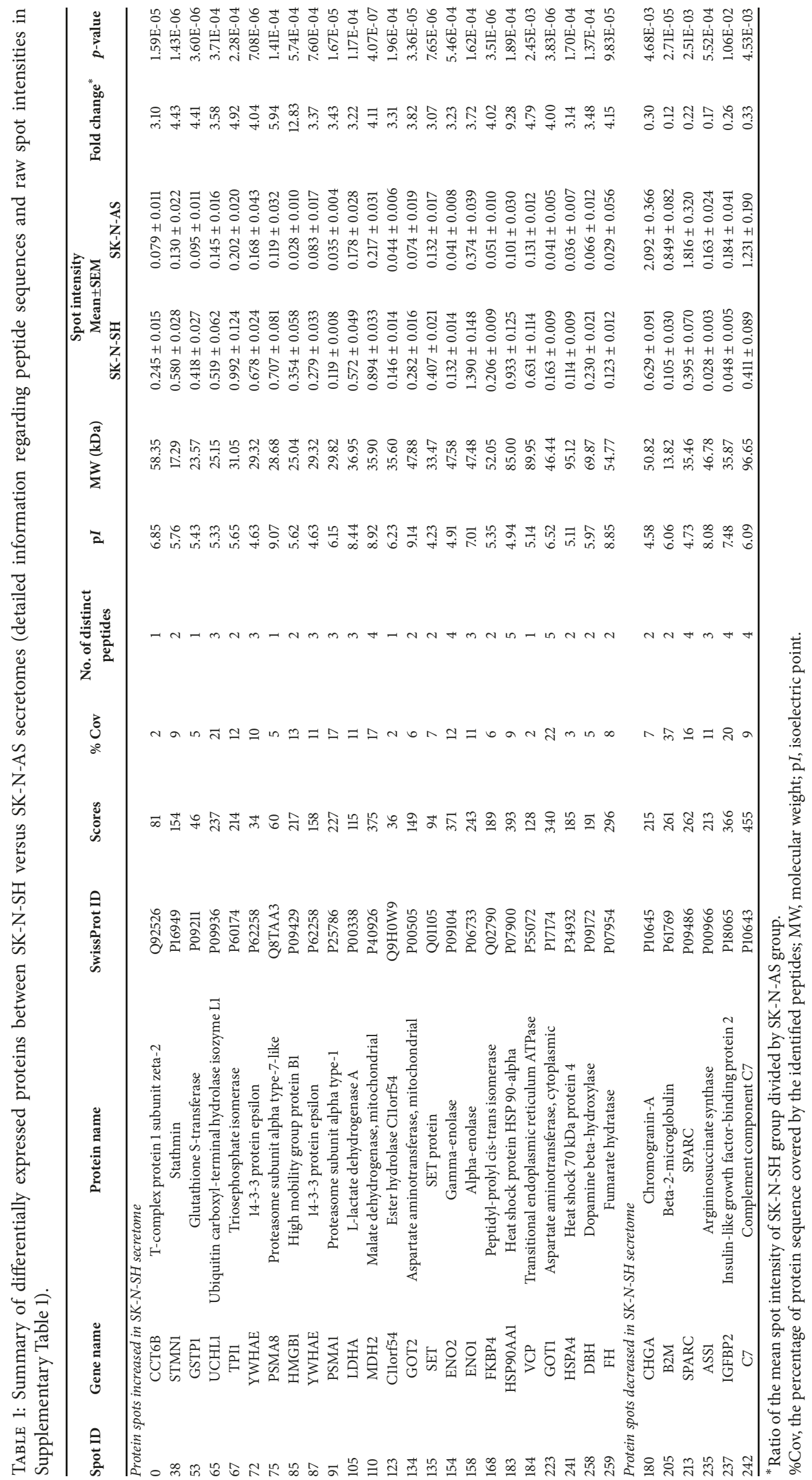




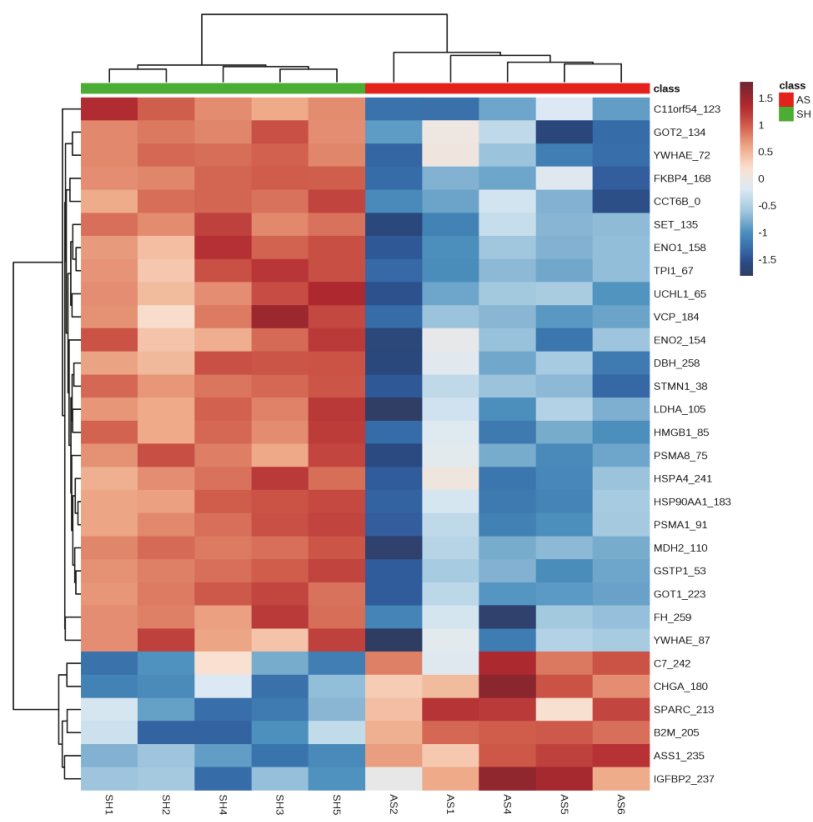

(a)

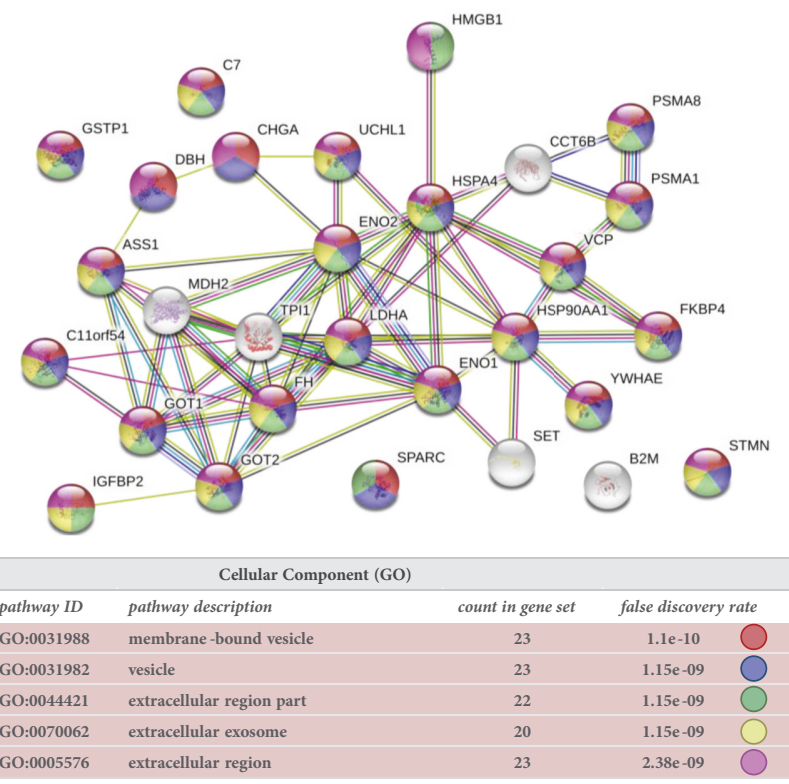

(b)

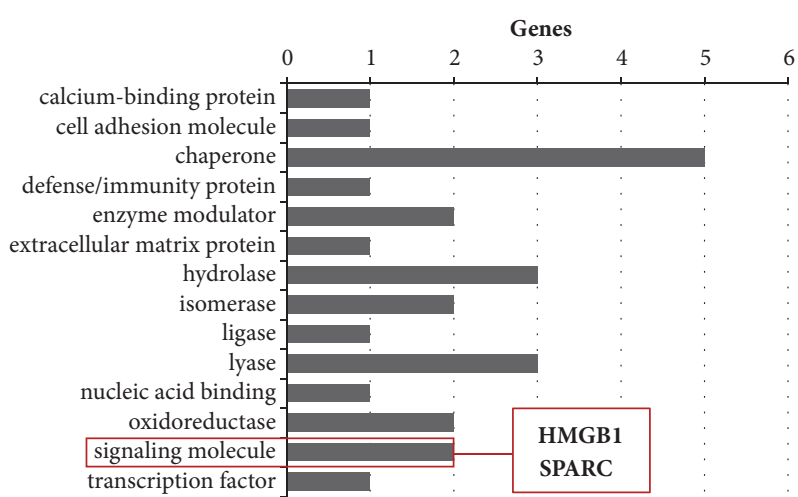

(c)

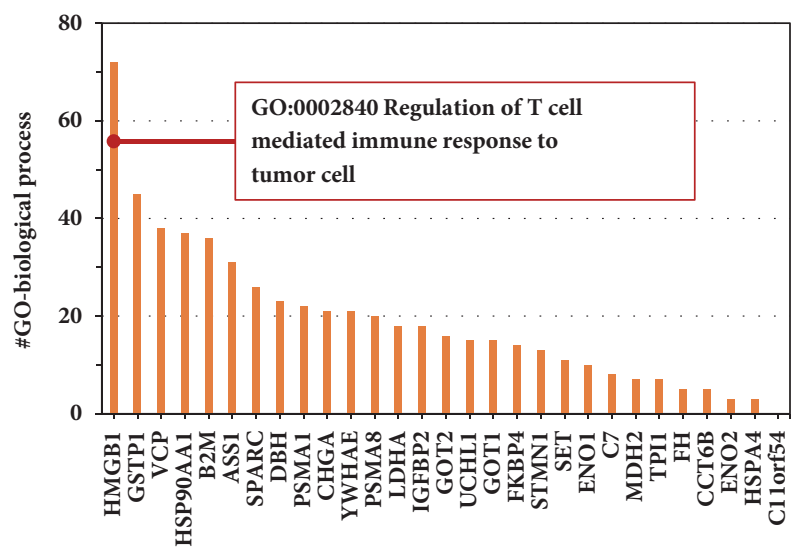

(d)

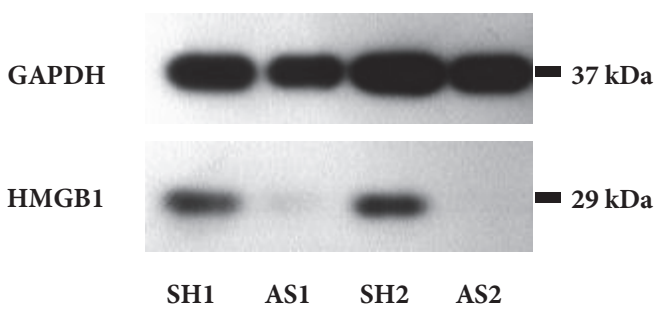

(e)

FIGURE 3: Protein bioinformatics and validation of HMGB1 expression. (a) A self-organized heatmap shows the relative expressional data of 29 significantly altered proteins (labeled by gene name with spot ID) in the secretomes of SK-N-SH (SH) versus SH-N-AS (AS) ( $\mathrm{n}=5$ per group). (b) STRING protein network with GO-cellular component analysis showed that 24 out of 29 proteins (colored nodes) were annotated as proteins in extracellular compartments (details in Supplementary Table 2). Gray nodes represented nonsecretory proteins. (c) Panther protein classification suggested that only 2 altered proteins functioned as the signaling molecules, in which one of them was HMGB1 (details in Supplementary Table 3). (d) David annotation of GO-term biological process showed that HMGB1 play multifunctional roles, one of which may involve in the regulation of $\mathrm{T}$ cell response to tumor cells (details in Supplementary Table 4). (e) HMGB1 levels in the SK-N-SH (SH) and SK-N-AS (AS) secretomes were validated by Western blot analysis in duplicate. GAPDH served as a loading control.

the SK-N-SH secretomes play a major role in Treg differentiation, neutralization studies using anti-HMGB1 mAb were performed in the coculture and supernatant treatment models (Figure 4(a)). Consistent with previous data (Figure 1(d)), $\mathrm{CD} 4^{+} \mathrm{CD} 25^{+}$Foxp $^{+}$Treg differentiation was observed after
PBMCs coculture with SK-N-SH NB cells for 4 days (Figure 4(a), left panel, and Figure 4(b)). Interestingly, addition of anti-HMGB1 mAb to the coculture suppressed Treg differentiation in a dose-dependent manner (Figure 4(b)). This result suggested that HMGB1 was an important factor for 


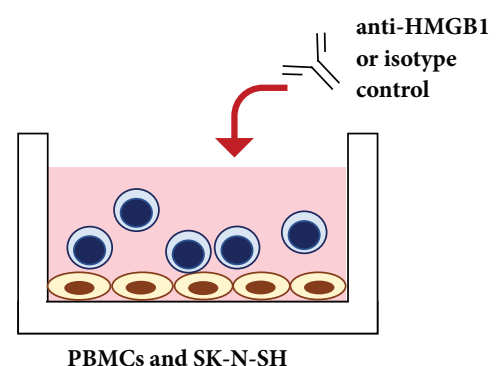

co-culture

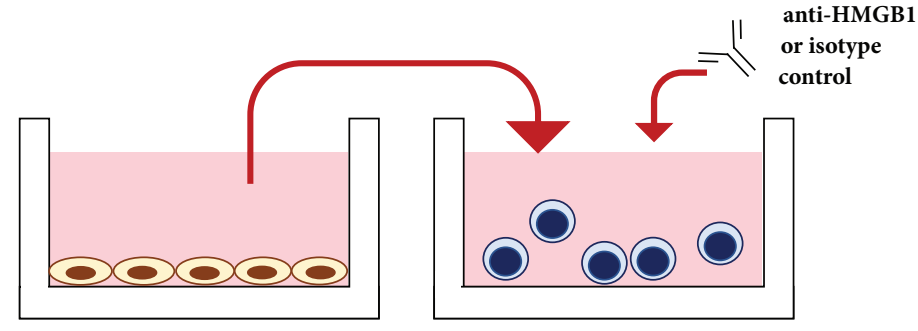

SK-N-SH supernatant treatment to PBMCs

PBMCs

SK-N-SH cells

(a)

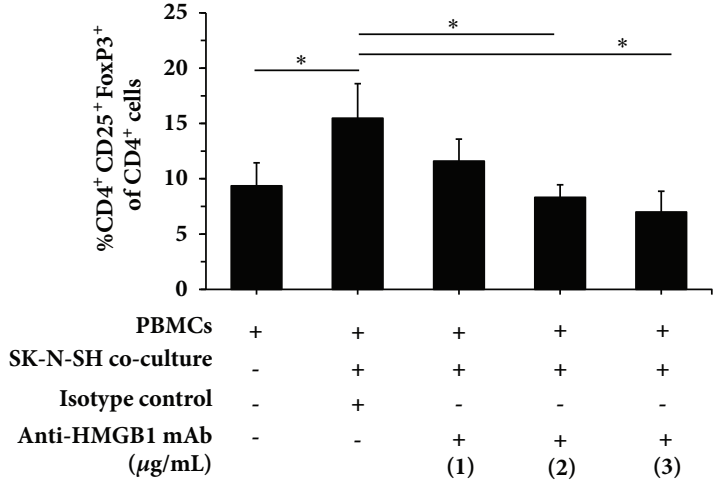

(b)

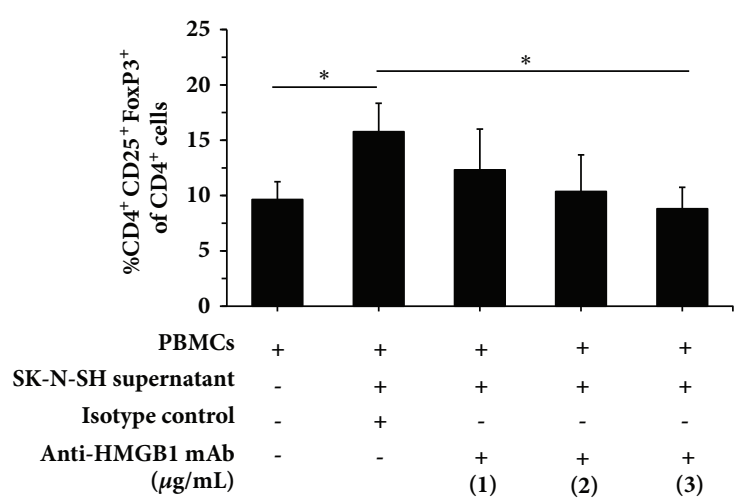

(c)

FIGURE 4: Neutralization by anti-HMGB1 inhibits Treg differentiation in a dose-dependent fashion. (a) Schematic diagram represents HMGB1 neutralization in the coculture (left panel) and the supernatant treatment (right panel) models. (b) The frequency of CD $4^{+} \mathrm{CD} 25^{+}$Foxp $3^{+}$Tregs of $\mathrm{CD}^{+} \mathrm{T}$ cells after PBMCs and SK-N-SH cell coculture in the presence of anti-HMGB1 mAb (1, 2, or $\left.3 \mu \mathrm{g} / \mathrm{mL}\right)$. (c) The frequency of $\mathrm{CD} 4^{+} \mathrm{CD} 25^{+} \mathrm{Foxp}^{+}$Tregs of $\mathrm{CD} 4^{+} \mathrm{T}$ cells after the SK-N-SH supernatant treatment to PBMCs in the presence of anti-HMGB1 mAb $(1,2$, or $3 \mu \mathrm{g} / \mathrm{mL}$ ). Cells were collected on day 4 for Treg enumeration. Baseline Treg levels were measured in PBMCs culture using complete media at day 4 . Goat $\operatorname{IgG}(1 \mu \mathrm{g} / \mathrm{mL})$ was served as the isotype control. PBMCs were obtained from 3 healthy individuals. Values represent mean \pm SD of 3 independent experiments $\left({ }^{*} p<0.05\right)$.

Treg differentiation in NB TME constituting both cancer cells and their secretomes.

To further validate that only secretory HMGB1 but not costimulatory factors of cell-to-cell contact was required for Treg differentiation, PBMCs were then solely treated with SK-N-SH culture supernatant (Figure 4(a), right panel) and subsequently subjected to the neutralization assay. As expected, SK-N-SH supernatant treatment resulted in the increased $\mathrm{CD} 4{ }^{+} \mathrm{CD} 25^{+}$Foxp $3^{+}$Treg levels (Figure 4(c)) as similar as those of the coculture experiment (Figure 4(b)). Again, anti-HMGB1 mAb neutralization could suppress Treg differentiation in dose-dependency (Figure 4(c)). This finding demonstrated that secretory HMGB1 play a central role of Treg differentiation in NB TME.

3.5. Integrated Analysis Showed HMGB1 Overexpression in Neuroblastoma Tumors Associated with Poor Clinical Outcomes. From a clinical standpoint, it is very interesting to address whether or not HMGB1 is overexpressed in patient tumors, and if so, that correlates at what extent to clinical outcomes. To gain some insight into the clinical relevance of HMGB1, we accessed the NCBI GEO database, accession GSE49711 (the MAQC-III/SEQC study) [28, 29], to adopt clinical and gene expression data of 498 pediatric neuroblastomas, focusing on determining the effects of HMGB1 expression in patient tumors associated with clinical outcomes (details in the Materials and Methods). Table 2 showed patient characteristics and numbers of patients with HMGB1 mRNA alterations in tumors. Overall, the MAQC-III/SEQC cohort covered the entire spectrum of the disease [29]. Patients with HMGB1-overexpressed tumors presented up to $11 \%$ of all cases (55/498) in this cohort.

Association analysis was performed to elucidate the effects of HMGB1 overexpression in NB tumors on clinical outcomes. Interestingly, the patients with HMGB1 mRNA upregulation in tumors showed significant associations with the occurrence of events including progression, relapse or death (adjusted OR of $2.78,95 \%$ CI of $1.49-5.22 ; p=0.001$ ), and the occurrence of death from disease (adjusted OR of $2.19,95 \%$ CI of $1.13-4.26 ; p=0.023$ ), whereas the patients 
TABLE 2: Patient characteristics of 498 pediatric neuroblastomas adopted from the MAQC-III/SEQC study. Data presented as number (\%).

\begin{tabular}{|c|c|}
\hline & $\begin{array}{c}\text { MAQC-III/SEQC }^{a} \\
\mathrm{n}=498\end{array}$ \\
\hline \multicolumn{2}{|l|}{$\overline{\text { Age }}$} \\
\hline$<18$ months & $300(60.2)$ \\
\hline$\geq 18$ months & $198(39.8)$ \\
\hline \multicolumn{2}{|l|}{ Gender } \\
\hline Male & $287(57.6)$ \\
\hline Female & $211(42.4)$ \\
\hline \multicolumn{2}{|l|}{ INSS stage } \\
\hline $4 \mathrm{~S}$ & $53(10.6)$ \\
\hline 1 & $121(24.3)$ \\
\hline 2 & $78(15.7)$ \\
\hline 3 & $63(12.7)$ \\
\hline 4 & $183(36.8)$ \\
\hline \multicolumn{2}{|l|}{ Risk group } \\
\hline High risk & $176(35.3)$ \\
\hline \multicolumn{2}{|l|}{ MYCN status } \\
\hline Amplification & $92(18.5)$ \\
\hline Non-amplification & $401(80.5)$ \\
\hline Unknown & $5(1.0)$ \\
\hline \multicolumn{2}{|c|}{ HMGB1 mRNA expression ${ }^{b}$} \\
\hline Upregulation & $55(11.0)$ \\
\hline Downregulation & $50(10.0)$ \\
\hline No alteration & $393(79.0)$ \\
\hline
\end{tabular}

with HMGB1 mRNA downregulation in tumors had no association to unfavorable outcomes (Figure 5). Direct evidence of clinical associations, together with the findings of Treg differentiation due to high HMGB1 levels in NB TME in vitro, supported future investigations which aim to mitigate HMGB1 overexpression in tumors and/or to neutralize secretory HMGB1 in NB TME.

\section{Discussion}

During the past few decades, a large body of evidence has uncovered a pivotal role of the TME in solid tumors in escaping host immunity. However, limited data are available on the significance of circulating and intratumoral Tregs in NB patients. Morandi et al. (2015) reported higher percentage of circulating Tregs in metastatic patients than healthy controls [17]. The finding is in accordance with those demonstrated by Tilak et al. (2014) [16]. Despite ambiguous data on the Treg frequency and the clinical outcome, depletion of these immunosuppressive $\mathrm{T}$ cells seems to be beneficial in cancer treatment $[20,32-34]$. Transient depletion of either $\mathrm{CD} 4^{+}$or $\mathrm{CD} 25^{+} \mathrm{T}$ cells has been shown to enhance immunotherapy and antitumor immunity in murine NB models [19, 20, 35, 36].

In the present study, we sought to gain a better understanding of NB-derived secretomes involved in promoting Treg immunophenotype. A study by Carlson et al. (2013) compared CD25 surface expression of peripheral blood lymphocytes (PBLs) and tumor-associated lymphocytes (TALs) from NB patients and found a larger proportion of $\mathrm{T}$ cells expressing CD25 in autologous TALs indicating that the NB microenvironment can promote $\mathrm{T}$ cell activation [37]. The same group also reported CD25 upregulation when PBLs were cultured in the presence of autologous tumor cells. Consistent with the previous study, the coculture experiments in our study demonstrated that CD25 expression was upregulated when PBMCs were cultured with NB cells (Figure 1(c)); thus the TME is permissive for $\mathrm{T}$ cell activation. However, Carlson et al. (2013) did not find Foxp3 upregulation in TALs as compared to PBLs [37]. In contrast, we found that one NB cell line, SK-N-SH, exhibited an ability to induce Treg differentiation, while the other two cell lines, SK-NAS and SH-SY5Y, did not share this property. One possible explanation for this discrepancy may be attributable to a wide diversity of genetic mutations of NB tumors resulting in different immunological signature. The influence of the TME on Treg induction has been reported in other type of solid tumors such as hepatocellular carcinoma [38] and ovarian [39]. For example, coculture $\mathrm{CD}^{+}$cells with ovarian cancer cell line can induce $\mathrm{CD} 8^{+}$Tregs that can inhibit naïve $\mathrm{CD} 4^{+}$ $\mathrm{T}$ cell proliferation [39].

Tumor secreted proteins play important roles in cell-tocell communication and modulating host immune response in the TME niche. Up to date, several soluble factors which confer immunomodulatory properties have been identified. Based on the previous studies [30,31], HMGB1 is a potential candidate as an immune suppressive factor in our study. HMGB1 is a member of the danger associated molecular patterns (DAMPs) family that has divergent biological functions including inflammation, autophagy/apoptosis, promotes tumor cell survival, and mediates immune responses. The protein can engage with several receptors including advanced glycation end products (RAGE) and Toll-like receptors (TLRs) [40]. Extracellular HMGB1 is released by both cancer and infiltrating immune cells within the inflammatory TME. It can be found in the nucleus, the cytoplasm, on the cell surface, and the extracellular environment [41]. HMGB1 has been shown to play a role in both immunosuppressive and immune-activation properties [40]. As an immune-activating factor, HMGB1 released from necrotic tumor cells binds to Toll-like receptor 4 (TLR4) expressed by dendritic cells (DCs) to promote antigen presentation and also activates macrophage TNF release and thus activates antitumor immunity [42, 43]. Conversely, tumorderived HMGB1 may favor tumor progression and suppress antitumor immunity by promoting IL-10 production in Tregs through RAGE [30, 31]. HMGB1 is expressed in various cancers and is associated with cancer progression [44]. The protein is associated with tumor growth and chemoresistance in NB $[45,46]$. HMGB1 overexpression using a lentivirus in SH-SY5Y cells was found to promote cell growth and 


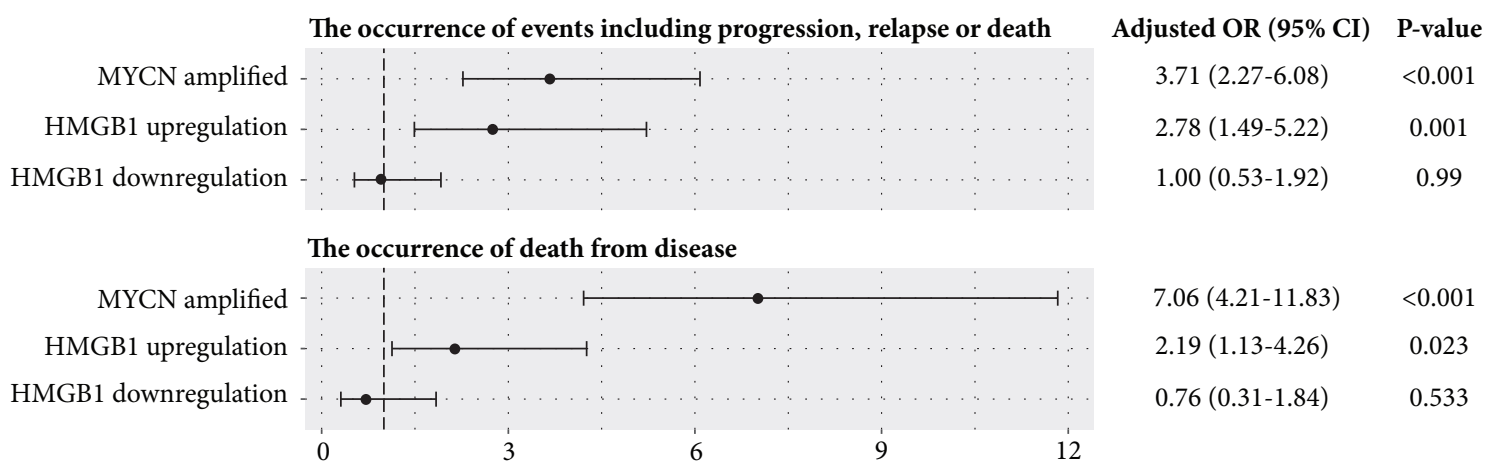

FIGURE 5: Association between HMGB1 expression in tumors and clinical outcomes in 498 neuroblastoma patients. Forest plot demonstrated that HMGB1 mRNA upregulation was an independent risk of poor clinical outcomes including disease progression, relapse, or death. MYCN amplification, a strong prognostic factor of neuroblastoma, was included in the model to adjust the contributed risk of HMGB1 mRNA alterations. Upregulation and downregulation of HMGB1 mRNA were defined as the z-score $>1.25$ and $<-1.25$, respectively. Data were made available in Supplementary Table 5.

migratory ability [46]. Another major role of HMGB1 is to function as a mediator of autophagy, which contributes to chemoresistance [41, 46, 47]. HMGB1 expression in cancer cells was upregulated following exposure to anticancer agents resulting in translocation of the protein to the cytoplasm $[46,47]$. Cytosolic HMGB1 competes with Bcl-2 in binding with Beclin 1 leading to the formation of autophagosomes $[47,48]$. Nonetheless, the role of HMGB1 in Tregs is still controversial. Preincubation of Tregs with HMGB1 has been found to reduce their inhibitory function and IL-10 secretion [49], while the other group demonstrated increased suppressive function and prolong survival of Tregs after HMGB1 stimulation [31].

Despite the findings from previous studies, the role of NBderived HMGB1 in promoting Treg differentiation has never been elucidated. The observations in this study suggested that NB-derived secretory HMGB1 enhance Treg differentiation by upregulating Foxp3, the transcription factor that dominantly controls Treg suppressive capacity [7], in activated $\mathrm{CD}^{+} \mathrm{CD} 25^{+} \mathrm{T}$ cells (Figures $1(\mathrm{~b})$ and $1(\mathrm{c})$ ). Inhibition of HMGB1 by the specific antibody significantly reduced the number of $\mathrm{CD} 4^{+} \mathrm{CD} 25^{+}$Foxp $3^{+}$Tregs in the dose-dependent manner (Figures 4(b) and 4(c)). By the fact that up to $11 \%$ of NB patients had HMGB1 overexpression in tumors (Table 2) and this was associated with the increased risk of adverse events including tumor progression, relapse, or death (Figure 5), antitumor immunity mediated by HMGB1induced Treg differentiation may be responsible for unfavorable outcomes. This finding has a translational potential, where HMGB1 in the NB TME is investigated as a therapeutic targeting of Tregs to improve antitumor immunity against NB in the future.

There are a number of limitations in our study. We did not examine the suppressive function of Tregs in the presence of NB cells. In addition, the mechanism of HMGB1-mediated Treg differentiation in our study including receptors and downstream signaling pathways remains to be elucidated. The limitation in the nature of the in vitro system should also be taken into account as we neglected the interaction between other cell types and tumor cells present in the in vivo environment. Furthermore, we did not examine the function of other significant proteins in SK-N-SH secretomes. Besides these limitations, our data showed that HMGB1 secreting NB cells could induce Treg differentiation in vitro and thus may serve as a potential therapeutic target in cancer immunotherapy.

\section{Conclusions}

In conclusion, our data demonstrate that the NB microenvironment is permissive of $\mathrm{T}$ lymphocyte activation and HMGB1 secreting NB cells can promote Treg differentiation. We propose HMGB1 as the major contributor of Treg differentiation in the NB TME. Further studies focusing on HMGB1-mediated Treg differentiation are warranted to mitigate immunosuppressive microenvironment which eventually improve the efficacy of NB immunotherapy.

\section{Data Availability}

The proteomic data and bioinformatic results used to support the findings of this study were included within the Supplementary Information. Clinical and RNA-seq datasets are accessible at the NCBI GEO database [28], accession GSE49711 [29].

\section{Conflicts of Interest}

The authors declare no conflicts of interest.

\section{Authors' Contributions}

Somchai Chutipongtanate initiated the ideation. Thitinee Vanichapol and Somchai Chutipongtanate developed the design. Suradej Hongeng made recommendations on the study design. Thitinee Vanichapol performed most experiments. Wararat Chiangjong performed mass spectrometry. Jirawan Panachan performed Western blot analysis. Thitinee Vanichapol, Wararat Chiangjong, Jirawan Panachan, Suradej 
Hongeng, and Somchai Chutipongtanate analyzed the data. Thitinee Vanichapol and Somchai Chutipongtanate prepared figures and tables. Thitinee Vanichapol wrote the manuscript. Wararat Chiangjong, Jirawan Panachan, Usanarat Anurathapan, Suradej Hongeng, and Somchai Chutipongtanate revised the manuscript. Suradej Hongeng supported the overall research strategy. Somchai Chutipongtanate finalized the manuscript. All authors read and approved the manuscript to be published.

\section{Acknowledgments}

We thank Paisan Jitthrontham, Research Center, Faculty of Medicine Ramathibodi Hospital, Mahidol University, for technical assistance. This work was supported by the Research Chair Grant from National Science and Technology Development Agency, Thailand (FDA-CO-2559-3325-TH to Suradej Hongeng). Somchai Chutipongtanate was supported by Faculty's Staff Development Program of Faculty of Medicine Ramathibodi Hospital, Mahidol University, and the National Research Council of Thailand and the National Science Technology and Innovation Policy Office, Thailand, for his research activities.

\section{Supplementary Materials}

Supplementary Table 1: detailed information of protein identities, peptide sequences, and spot intensities of differentially expressed proteins between SK-N-SH and SK-NAS secretomes. Supplementary Table 2: STRING with GOterm cellular component analysis of the significant proteins. Supplementary Table 3: panther protein classification of the significant proteins. Supplementary Table 4: GO-term biological process of the significant proteins using David functional annotation. Supplementary Table 5: detailed information of patient characteristics, clinical outcomes, and HMGB1 mRNA expression in 498 pediatric neuroblastomas adopted from the NCBI GEO database, accession GSE49711. (Supplementary Materials)

\section{References}

[1] M. R. Esposito, S. Aveic, A. Seydel, and G. P. Tonini, "Neuroblastoma treatment in the post-genomic era," Journal of Biomedical Science, vol. 24, no. 1, article 14, 2017.

[2] A. Heczey and C. U. Louis, "Advances in chimeric antigen receptor immunotherapy for neuroblastoma," Discovery Medicine, vol. 16, no. 90, pp. 287-294, 2013.

[3] I. Scarfò and M. V. Maus, "Current approaches to increase CAR T cell potency in solid tumors: Targeting the tumor microenvironment," Journal for Immuno Therapy of Cancer, vol. 5, no. 28, 2017.

[4] T. Vanichapol, S. Chutipongtanate, U. Anurathapan, and S. Hongeng, "Immune escape mechanisms and future prospects for immunotherapy in neuroblastoma," BioMed Research International, vol. 2018, Article ID 1812535, 11 pages, 2018.

[5] X. Cao, "Regulatory T cells and immune tolerance to tumors," Immunologic Research, vol. 46, no. 1-3, pp. 79-93, 2010.
[6] A. Facciabene, G. T. Motz, and G. Coukos, "T-Regulatory cells: key players in tumor immune escape and angiogenesis," Cancer Research, vol. 72, no. 9, pp. 2162-2171, 2012.

[7] T. L. Whiteside, P. Schuler, and B. Schilling, "Induced and natural regulatory T cells in human cancer," Expert Opinion on Biological Therapy, vol. 12, no. 10, pp. 1383-1397, 2012.

[8] K. Oleinika, R. J. Nibbs, G. J. Graham, and A. R. Fraser, "Suppression, subversion and escape: The role of regulatory $\mathrm{T}$ cells in cancer progression," Clinical \& Experimental Immunology, vol. 171, no. 1, pp. 36-45, 2013.

[9] D. A. A. Vignali, L. W. Collison, and C. J. Workman, "How regulatory T cells work," Nature Reviews Immunology, vol. 8, no. 7, pp. 523-532, 2008.

[10] B. Chaudhary and E. Elkord, "Regulatory T cells in the tumor microenvironment and cancer progression: Role and therapeutic targeting," Vaccines, vol. 4, no. 3, 2016.

[11] P. Xu, W. Fan, Z. Zhang et al., "The Clinicopathological and Prognostic Implications of FoxP3+ Regulatory T Cells in Patients with Colorectal Cancer: A Meta-Analysis," Frontiers in Physiology, vol. 8, 2017.

[12] F. Ihara, D. Sakurai, A. Horinaka et al., "CD45RA-Foxp3high regulatory $\mathrm{T}$ cells have a negative impact on the clinical outcome of head and neck squamous cell carcinoma," Cancer Immunology, Immunotherapy, vol. 66, no. 10, pp. 1275-1285, 2017.

[13] G. J. Bates, S. B. Fox, C. Han, R. D. Leek, J. F. Garcia, A. L. Harris et al., "Quantification of regulatory $\mathrm{T}$ cells enables the identification of high-risk breast cancer patients and those at risk of late relapse," Journal of Clinical Oncology, vol. 24, no. 34, pp. 5373-5380, 2006.

[14] N. Hiraoka, K. Onozato, T. Kosuge, and S. Hirohashi, "Prevalence of $\mathrm{FOXP}^{+}$regulatory $\mathrm{T}$ cells increases during the progression of pancreatic ductal adenocarcinoma and its premalignant lesions," Clinical Cancer Research, vol. 12, no. 18, pp. 54235434, 2006.

[15] Y. Que, W. Xiao, Y.-X. Guan et al., "PD-L1 expression is associated with FOXP3+ regulatory T-Cell infiltration of soft tissue sarcoma and poor patient prognosis," Journal of Cancer, vol. 8, no. 11, pp. 2018-2025, 2017.

[16] T. Tilak, S. Sherawat, S. Agarwala, R. Gupta, S. Vishnubhatla, and S. Bakhshi, "Circulating T-regulatory cells in neuroblastoma: a pilot prospective study," Pediatric Hematology and Oncology, vol. 31, no. 8, pp. 717-722, 2014.

[17] F. Morandi, M. Croce, G. Cangemi, S. Barco, V. Rigo, B. Carlini et al., "IL-10 and ARG-1 concentrations in bone marrow and peripheral blood of metastatic neuroblastoma patients do not associate with clinical outcome," Journal of Immunology Research, vol. 2015, Article ID 718975, 9 pages, 2015.

[18] B. D. Johnson, W. Jing, and R. J. Orentas, “CD25+ regulatory T cell inhibition enhances vaccine-induced immunity to neuroblastoma," Journal of Immunotherapy, vol. 30, no. 2, pp. 203-214, 2007.

[19] M. Croce, M. V. Corrias, A. M. Orengo et al., "Transient depletion of $\mathrm{CD}^{+}{ }^{+} \mathrm{T}$ cells augments IL-21-based immunotherapy of disseminated neuroblastoma in syngeneic mice," International Journal of Cancer, vol. 127, no. 5, pp. 1141-1150, 2010.

[20] W. Jing, X. Yan, W. H. D. Hallett, J. A. Gershan, and B. D. Johnson, "Depletion of CD25 $5^{+} \mathrm{T}$ cells from hematopoietic stem cell grafts increases posttransplantation vaccine-induced immunity to neuroblastoma," Blood, vol. 117, no. 25, pp. 69526962, 2011. 
[21] J. Zheng, P. Liu, and X. Yang, "YB-1 immunization combined with regulatory $\mathrm{T}$-cell depletion induces specific T-cell responses that protect against neuroblastoma in the early stage," Acta Biochimica et Biophysica Sinica, vol. 44, no. 12, pp. 10061014, 2012.

[22] J. L. Paltridge, L. Belle, and Y. Khew-Goodall, "The secretome in cancer progression," Biochimica et Biophysica Acta, vol. 1834, no. 11, pp. 2233-2241, 2013.

[23] W. Rozek, M. Kwasnik, J. Debski, and J. F. Zmudzinski, "Mass spectrometry identification of granins and other proteins secreted by neuroblastoma cells," Tumor Biology, vol. 34, no. 3, pp. 1773-1781, 2013.

[24] J. G. Xia, N. Psychogios, N. Young, and D. S. Wishart, "MetaboAnalyst: a web server for metabolomic data analysis and interpretation," Nucleic Acids Research, vol. 37, no. 2, pp. W652-W660, 2009.

[25] A. Franceschini, D. Szklarczyk, S. Frankild et al., "STRING v9.1: protein-protein interaction networks, with increased coverage and integration," Nucleic Acids Research, vol. 41, no. 1, pp. D808D815, 2013.

[26] H. Mi, A. Muruganujan, and P. D. Thomas, "PANTHER in 2013: modeling the evolution of gene function, and other gene attributes, in the context of phylogenetic trees," Nucleic Acids Research, vol. 41, no. 1, pp. D377-D386, 2013.

[27] D. W. Huang, B. T. Sherman, and R. A. Lempicki, "Systematic and integrative analysis of large gene lists using DAVID bioinformatics resources," Nature Protocols, vol. 4, no. 1, pp. 44-57, 2009.

[28] T. Barrett, S. E. Wilhite, P. Ledoux et al., "NCBI GEO: archive for functional genomics data sets-update," Nucleic Acids Research, vol. 41, no. 1, pp. D991-D995, 2013.

[29] W. Zhang, Y. Yu, F. Hertwig, J. Thierry-Mieg, D. Thierry-Mieg et al., "Comparison of RNA-seq and microarray-based models for clinical endpoint prediction," Genome Biology, vol. 25, no. 16, 2015.

[30] Z. Liu, L. D. Falo Jr., and Z. You, "Knockdown of HMGB1 in tumor cells attenuates their ability to induce regulatory $\mathrm{T}$ cells and uncovers naturally acquired CD8 T cell-dependent antitumor immunity," The Journal of Immunology, vol. 187, no. 1, pp. 118-125, 2011.

[31] C. A. Wild, C. Bergmann, G. Fritz et al., "HMGB1 conveys immunosuppressive characteristics on regulatory and conventional T cells," International Immunology, vol. 24, no. 8, pp. 485494, 2012.

[32] Y.-C. Shen, A. Ghasemzadeh, C. M. Kochel et al., "Combining intratumoral Treg depletion with androgen deprivation therapy (ADT): preclinical activity in the Myc-CaP model," Prostate Cancer and Prostatic Diseases, vol. 21, no. 1, pp. 113-125, 2018.

[33] S. A. Fisher, W. J. Aston, J. Chee et al., "Transient Treg depletion enhances therapeutic anti-cancer vaccination," Immunity, Inflammation and Disease, vol. 5, no. 1, pp. 16-28, 2017.

[34] M. Fogg, J. R. Murphy, J. Lorch, M. Posner, and F. Wang, "Therapeutic targeting of regulatory $\mathrm{T}$ cells enhances tumorspecific CD8+ T cell responses in Epstein-Barr virus associated nasopharyngeal carcinoma," Virology, vol. 441, no. 2, pp. 107113, 2013.

[35] V. Rigo, M. V. Corrias, A. M. Orengo et al., "Recombinant IL-21 and anti-CD4 antibodies cooperate in syngeneic neuroblastoma immunotherapy and mediate long-lasting immunity," Cancer Immunology, Immunotherapy, vol. 63, no. 5, pp. 501-511, 2014.

[36] W. Jing, J. A. Gershan, and B. D. Johnson, "Depletion of CD4 T cells enhances immunotherapy for neuroblastoma after syngeneic HSCT but compromises development of antitumor immune memory," Blood, vol. 113, no. 18, pp. 4449-4457, 2009.

[37] L. Carlson, A. De Geer, B. Sveinbjørnsson et al., "The microenvironment of human neuroblastoma supports the activation of tumor-associated T lymphocytes," OncoImmunology, vol. 2, no. 3, Article ID e23618, 2014.

[38] Y. Fan, Y. Gao, J. Rao, K. Wang, F. Zhang, and C. Zhang, "YAP1 Promotes Tregs Differentiation in Hepatocellular Carcinoma by Enhancing TGFBR2 Transcription," Cellular Physiology and Biochemistry, vol. 41, no. 3, pp. 1189-1198, 2017.

[39] S. Zhang, X. Ke, S. Zeng et al., "Analysis of CD8+ Treg cells in patients with ovarian cancer: a possible mechanism for immune impairment," Cellular \& Molecular Immunology, vol. 12, no. 5, pp. 580-591, 2015.

[40] R. Kang, Q. Zhang, H. J. Zeh III, M. T. Lotze, and D. Tang, "HMGB1 in cancer: good, bad, or both?" Clinical Cancer Research, vol. 19, no. 15, pp. 4046-4057, 2013.

[41] V. Pistoia and A. Pezzolo, "Involvement of HMGB1 in resistance to tumor vessel-targeted, monoclonal antibody-based immunotherapy," Journal of Immunology Research, vol. 2016, Article ID 3142365, 7 pages, 2016.

[42] L. Apetoh, F. Ghiringhelli, A. Tesniere et al., “Toll-like receptor 4-dependent contribution of the immune system to anticancer chemotherapy and radiotherapy," Nature Medicine, vol. 13, no. 9, pp. 1050-1059, 2007.

[43] H. Yang, H. S. Hreggvidsdottir, K. Palmblad et al., "A critical cysteine is required for HMGB1 binding to toll-like receptor 4 and activation of macrophage cytokine release," Proceedings of the National Acadamy of Sciences of the United States of America, vol. 107, no. 26, pp. 11942-11947, 2010.

[44] S.-J. He, J. Cheng, X. Feng, Y. Yu, L. Tian, and Q. Huang, "The dual role and therapeutic potential of high-mobility group box 1 in cancer," Oncotarget , vol. 8, no. 38, pp. 64534-64550, 2017.

[45] E. Mari, A. Zicari, F. Fico, I. Massimi, L. Martina, and S. Mardente, "Action of HMGB1 on miR-221/222 cluster in neuroblastoma cell lines," Oncology Letters, vol. 12, no. 3, pp. 21332138, 2016.

[46] L. Wang, H. Zhang, M. Sun, Z. Yin, and J. Qian, "High mobility group box 1-mediated autophagy promotes neuroblastoma cell chemoresistance," Oncology Reports, vol. 34, no. 6, pp. 29692976, 2015.

[47] Y. Xiao, L. Sun, Y. Fu et al., "High mobility group box 1 promotes sorafenib resistance in HepG2 cells and in vivo," BMC Cancer, vol. 17, no. 1, 2017.

[48] R. Kang, K. M. Livesey, H. J. Zeh, M. T. Lotze, and D. Tang, "HMGB1: a novel Beclin 1-binding protein active in autophagy," Autophagy, vol. 6, no. 8, pp. 1209-1211, 2010.

[49] C. Luo, H. Liu, H. Wang, and J. Wang, "Toll-like receptor 4 signaling in high mobility group box-1 protein 1 mediated the suppression of regulatory T-cells," Medical Science Monitor, vol. 23, pp. 300-308, 2017. 


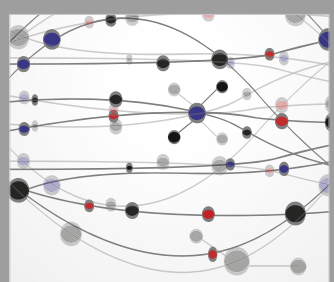

The Scientific World Journal
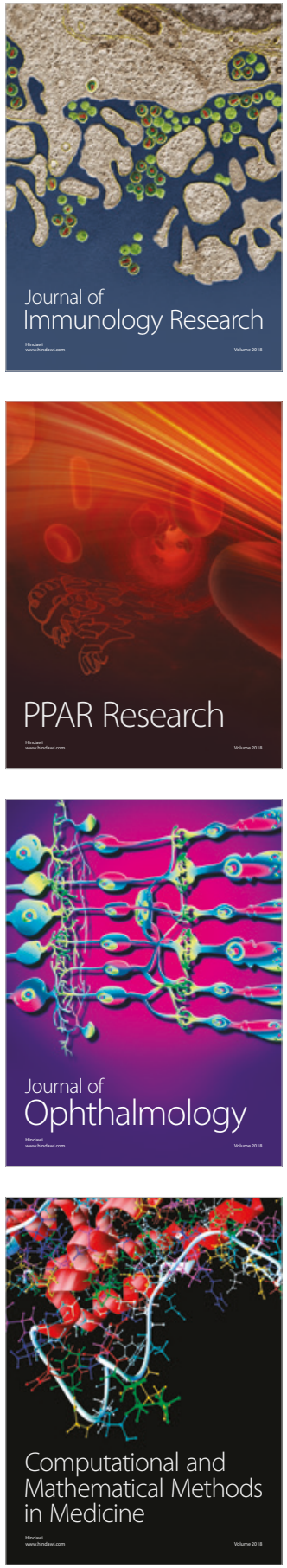

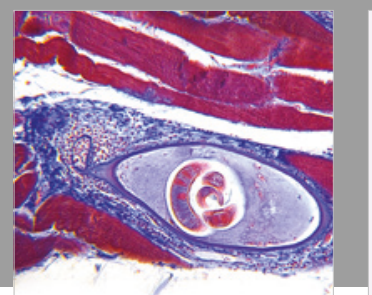

Gastroenterology Research and Practice

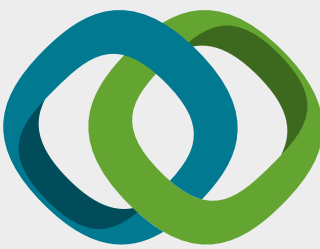

\section{Hindawi}

Submit your manuscripts at

www.hindawi.com
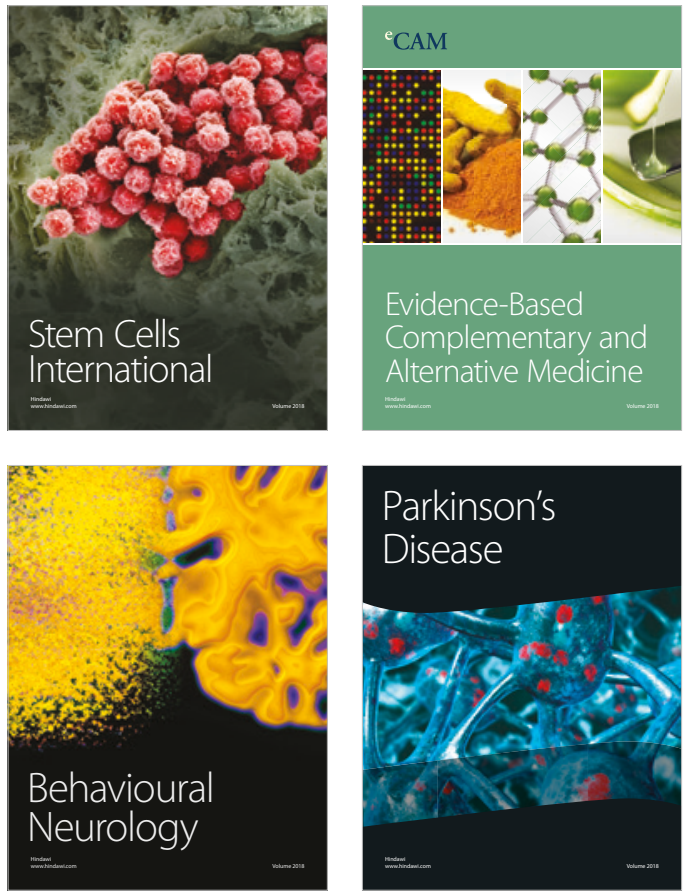

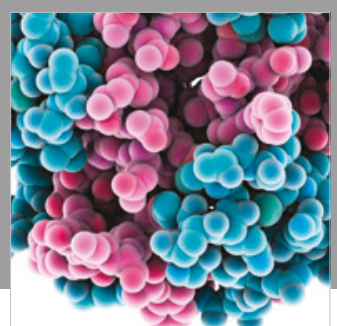

ournal of

Diabetes Research

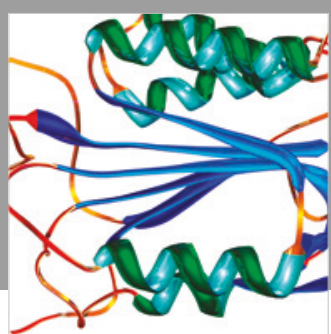

Disease Markers
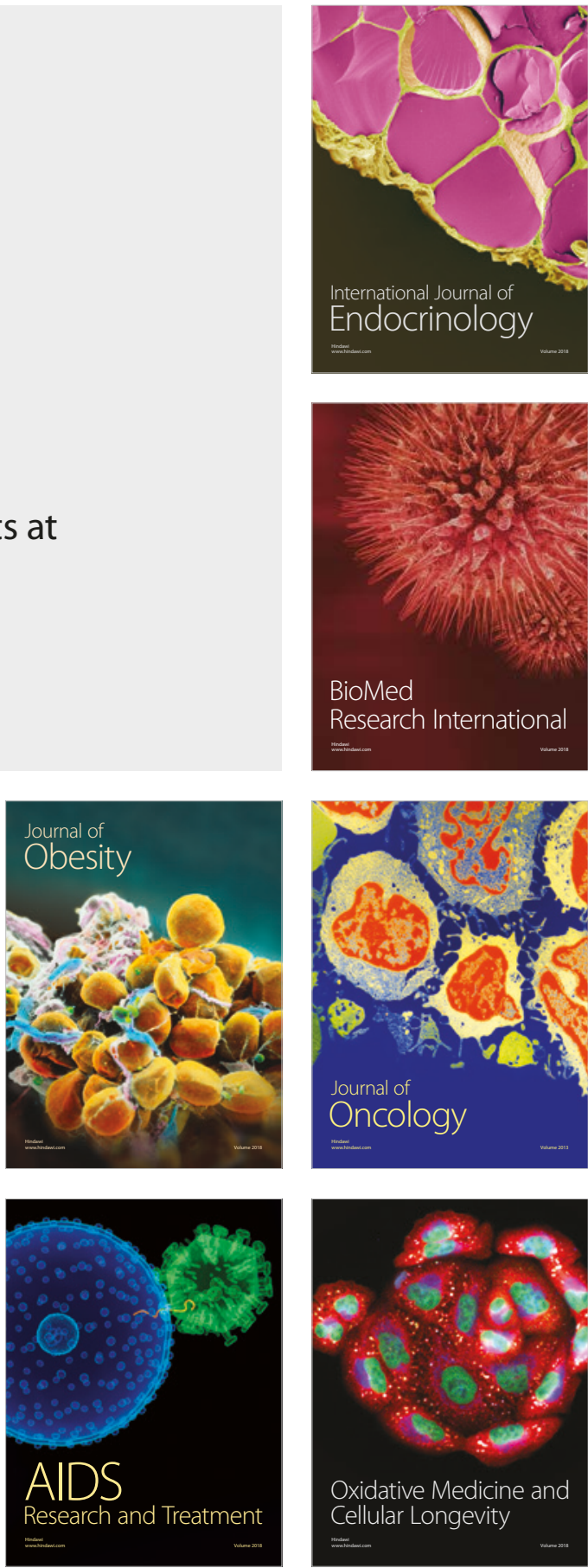